Supplement of Geosci. Model Dev., 13, 3299-3318, 2020

https://doi.org/10.5194/gmd-13-3299-2020-supplement

(C) Author(s) 2020. This work is distributed under

the Creative Commons Attribution 4.0 License.

(c) (1)

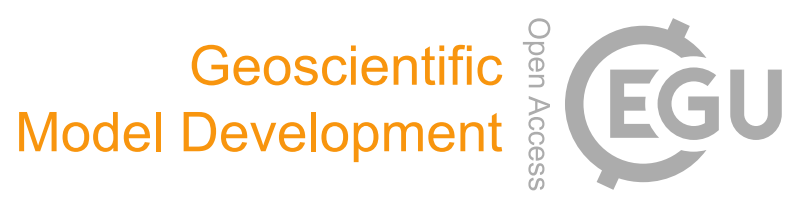

Supplement of

\title{
Quantitative assessment of fire and vegetation properties in simulations with fire-enabled vegetation models from the Fire Model Intercomparison Project
}

Stijn Hantson et al.

Correspondence to: Stijn Hantson (hantson.stijn@gmail.com)

The copyright of individual parts of the supplement might differ from the CC BY 4.0 License. 
Table S1: Full benchmarking table with all scores for each model, reference dataset and step considered.

\begin{tabular}{|c|c|c|c|c|c|c|c|c|c|c|c|c|c|}
\hline & nroduct & sten & mean & random & CIM & CLASS- & $\begin{array}{l}\text { JULES } \\
\text { JNFERNO }\end{array}$ & $\begin{array}{l}\text { JSBACH } \\
\text { SPITFIRE }\end{array}$ & LPJ-G & $\begin{array}{l}\text { LPJ-G } \\
\text { SPITFIRF }\end{array}$ & SIMFIRE & & ORCHIDEE \\
\hline \multicolumn{14}{|c|}{ burnt area } \\
\hline \multirow[t]{15}{*}{ spatial } & GFED4s & step1 & 1 & 1.07 & 0.60 & 0.81 & 0.63 & 0.67 & 0.73 & 0.84 & 0.83 & 0.69 & 0.71 \\
\hline & GFED4 & step1 & 1 & 1.14 & 0.81 & 1.06 & 0.77 & 0.88 & 0.71 & 0.98 & 0.98 & 0.78 & 0.82 \\
\hline & MCD45 & step1 & 1 & 1.07 & 0.68 & 0.94 & 0.68 & 0.74 & 0.72 & 0.93 & 1.00 & 0.71 & 0.81 \\
\hline & FireCCI4.0 & step1 & 1 & 1.13 & 0.75 & 0.83 & 0.78 & 0.91 & 0.70 & 0.90 & 1.01 & 0.80 & 0.81 \\
\hline & FireCCI5.1 & Step1 & 1 & 1.11 & 0.83 & 1.10 & 0.81 & 0.95 & 0.71 & 0.89 & 0.99 & 0.85 & 0.74 \\
\hline & GFED4s & step2 & 1 & 1.07 & 0.62 & 0.84 & 0.71 & 0.68 & 0.98 & 0.88 & 0.83 & 0.96 & 0.72 \\
\hline & GFED4 & step2 & 1 & 1.14 & 0.84 & 1.12 & 0.76 & 0.89 & 0.98 & 1.00 & 1.02 & 0.99 & 0.91 \\
\hline & MCD45 & step2 & 1 & 1.16 & 0.77 & 1.08 & 0.69 & 0.84 & 0.98 & 0.95 & 1.05 & 0.97 & 0.90 \\
\hline & FireCCI40 & step2 & 1 & 1.13 & 0.77 & 0.91 & 0.78 & 0.92 & 0.99 & 0.91 & 1.05 & 1.02 & 0.88 \\
\hline & FireCCI51 & Step2 & 1 & 1.11 & 0.85 & 1.14 & 0.84 & 0.96 & 0.98 & 0.90 & 1.01 & 1.02 & 0.77 \\
\hline & GFED4s & step3 & 1 & 1.07 & 0.63 & 0.79 & 0.72 & 0.70 & 1.06 & 0.94 & 0.88 & 1.00 & 0.72 \\
\hline & GFED4 & step3 & 1 & 1.14 & 0.80 & 0.93 & 0.85 & 0.86 & 1.08 & 0.98 & 0.88 & 1.07 & 0.71 \\
\hline & MCD45 & step3 & 1 & 1.16 & 0.65 & 0.81 & 0.72 & 0.69 & 1.12 & 0.93 & 0.92 & 1.02 & 0.70 \\
\hline & FireCCI40 & step3 & 1 & 1.13 & 0.77 & 0.98 & 0.89 & 0.92 & 1.09 & 0.93 & 0.97 & 1.13 & 0.73 \\
\hline & FireCCI51 & Step3 & 1 & 1.11 & 0.83 & 1.01 & 0.91 & 0.93 & 1.11 & 0.96 & 0.97 & 1.23 & 0.70 \\
\hline seasonal & GFED4s & step1 & 0.56 & 0.22 & 0.12 & 0.12 & 0.13 & 0.12 & & 0.31 & & & 0.31 \\
\hline \multirow[t]{3}{*}{ phase } & GFED4 & step1 & 0.49 & 0.47 & 0.34 & 0.35 & 0.41 & 0.42 & & 0.33 & & & 0.31 \\
\hline & MCD45 & step1 & 0.56 & 0.26 & 0.12 & 0.11 & 0.12 & 0.12 & & 0.30 & & & 0.30 \\
\hline & FireCCI51 & Step1 & 0.55 & 0.33 & 0.26 & 0.28 & 0.33 & 0.32 & & 0.32 & & & 0.31 \\
\hline seasonal & GFED4s & step1 & 1 & 1.36 & 1.09 & 1.22 & 1.74 & 1.37 & & 1.07 & & & 1.12 \\
\hline
\end{tabular}




\begin{tabular}{|c|c|c|c|c|c|c|c|c|c|c|c|c|c|}
\hline \multirow[t]{11}{*}{ concentration } & GFED4 & step1 & 1 & 1.35 & 1.37 & 1.56 & 2.49 & 1.83 & & 1.23 & & & 1.31 \\
\hline & MCD45 & step1 & 1 & 1.36 & 1.26 & 1.40 & 2.40 & 1.70 & & 1.15 & & & 1.23 \\
\hline & FireCCI51 & Step1 & 1 & 1.36 & 1.48 & 1.72 & 2.75 & 2.06 & & 1.26 & & & 1.45 \\
\hline & GFED4s & step2 & 1 & 1.36 & 1.07 & 1.20 & 1.14 & 1.28 & & 1.06 & & & 1.13 \\
\hline & GFED4 & step2 & 1 & 1.35 & 1.20 & 1.39 & 1.28 & 1.48 & & 1.20 & & & 1.23 \\
\hline & MCD45 & step2 & 1 & 1.36 & 1.18 & 1.31 & 1.29 & 1.49 & & 1.15 & & & 1.21 \\
\hline & FireCCI51 & Step2 & 1 & 1.36 & 1.27 & 1.53 & 1.38 & 1.64 & & 1.22 & & & 1.34 \\
\hline & GFED4s & step3 & 1 & 1.36 & 1.16 & 1.15 & 1.24 & 1.15 & & 1.13 & & & 1.22 \\
\hline & GFED4 & step3 & 1 & 1.35 & 1.19 & 1.12 & 1.25 & 1.11 & & 1.18 & & & 1.19 \\
\hline & MCD45 & step3 & 1 & 1.36 & 1.14 & 1.08 & 1.26 & 1.13 & & 1.12 & & & 1.20 \\
\hline & FireCCI51 & Step3 & 1 & 1.36 & 1.25 & 1.22 & 1.33 & 1.21 & & 1.20 & & & 1.27 \\
\hline \multirow[t]{4}{*}{ IAV } & GFED4s & step3 & 1 & 1.46 & 1.17 & 0.65 & 1.18 & 1.09 & 0.66 & 1.36 & 0.76 & 1.66 & 1.44 \\
\hline & GFED4 & step3 & 1 & 1.27 & 0.98 & 1.62 & 1.23 & 0.89 & 1.04 & 1.08 & 1.00 & 1.41 & 1.25 \\
\hline & MCD45 & step3 & 1 & 1.32 & 0.93 & 1.34 & 1.11 & 0.84 & 0.73 & 0.97 & 1.27 & 1.67 & 1.22 \\
\hline & FireCCI51 & step3 & 1 & 1.42 & 1.18 & 1.53 & 1.24 & 1.27 & 1.73 & 1.27 & 1.23 & 1.87 & 1.12 \\
\hline \multicolumn{14}{|l|}{ fire emission } \\
\hline \multirow[t]{3}{*}{ spatial } & GFAS & step1 & 1 & 1.08 & 0.72 & 1.12 & 0.74 & 0.81 & 0.75 & 0.94 & 0.95 & 0.64 & 1.11 \\
\hline & & step2 & 1 & 1.08 & 0.74 & 1.33 & 0.79 & 0.91 & 0.97 & 0.98 & 0.98 & 0.99 & 1.22 \\
\hline & & step3 & 1 & 1.08 & 0.78 & 0.85 & 0.73 & 0.74 & 1.13 & 1.03 & 0.91 & 1.06 & 0.86 \\
\hline $\begin{array}{l}\text { seasonal } \\
\text { phase }\end{array}$ & GFAS & step1 & 0.78 & 0.18 & 0.16 & 0.20 & 0.17 & 0.15 & & 0.37 & & & 0.34 \\
\hline seasonal & GFAS & step1 & 1 & 1.36 & 1.14 & 1.70 & 1.71 & 1.39 & & 1.21 & & & 1.15 \\
\hline \multirow[t]{2}{*}{ concentration } & & step2 & 1 & 1.36 & 1.11 & 1.29 & 1.21 & 1.28 & & 1.16 & & & 1.15 \\
\hline & & step3 & 1 & 1.36 & 1.20 & 1.22 & 1.30 & 1.17 & & 1.27 & & & 1.25 \\
\hline
\end{tabular}




\section{fire number}

spatial

$\begin{array}{lll}\text { step3 } & 1 & 1.19\end{array}$

0.96

\section{fire size}

spatial

Hantson

$\begin{array}{lll}\text { step3 } & 1 & 1.31\end{array}$

\section{GPP}

spatial

Luyssaert

$\begin{array}{lllll}\text { step1 } & 1 & 1.39 & 2.71 & 1.28 \\ \text { step2 } & 1 & 1.39 & 1.32 & 1.23 \\ \text { step3 } & 1 & 1.39 & 1.49 & 1.41\end{array}$

$\begin{array}{ll}1.39 & 1.48 \\ 1.33 & 1.15 \\ 1.46 & 1.39 \\ & \\ 0.49 & 0.72 \\ 0.51 & 0.62 \\ 0.39 & 0.42 \\ 0.19 & 0.23 \\ 0.59 & 0.65 \\ 0.70 & 0.76 \\ 1.14 & 1.21\end{array}$

2.45
1.13
1.41

1.01

spatial

Jung

$\begin{array}{lllll}\text { step1 } & 1 & 1.30 & 0.61 & 0.48\end{array}$

0.47

$\begin{array}{lllll}\text { step2 } & 1 & 1.30 & 0.61 & 0.50\end{array}$

0.46

$\begin{array}{lllll}\text { step3 } & 1 & 1.30 & 0.64 & 0.46\end{array}$

seasonal

Jung

$\begin{array}{lrrrrrr}\text { phase } & 0.42 & 0.65 & 0.18 & 0.23 & 0.19 & 0.23 \\ \text { step1 } & 1 & 1.65 & 0.60 & 0.63 & 0.59 & 0.65 \\ \text { step2 } & 1 & 1.65 & 0.69 & 0.74 & 0.70 & 0.76 \\ \text { step3 } & 1 & 1.65 & 1.08 & 1.19 & 1.14 & 1.21\end{array}$

NPP

\begin{tabular}{|c|c|c|c|c|c|c|c|c|c|c|c|c|c|}
\hline \multirow[t]{3}{*}{ spatial } & Michaletz & step1 & 1 & 1.39 & 1.27 & 0.99 & 1.16 & 1.12 & 1.48 & 1.60 & 1.55 & 1.03 & 1.28 \\
\hline & & step2 & 1 & 1.39 & 0.79 & 0.77 & 0.77 & 0.78 & 0.86 & 0.85 & 0.84 & 0.84 & 0.87 \\
\hline & & step3 & 1 & 1.39 & 0.82 & 0.79 & 0.77 & 0.75 & 0.96 & 0.86 & 0.89 & 0.88 & 0.99 \\
\hline \multirow[t]{2}{*}{ spatial } & Luyssaert & step1 & 1 & 1.33 & 0.88 & 1.34 & 0.62 & 1.90 & 0.77 & 0.81 & 0.74 & 1.11 & 0.78 \\
\hline & & step2 & 1 & 1.33 & 0.89 & 1.26 & 0.61 & 1.00 & 0.78 & 0.77 & 0.75 & 1.16 & 0.77 \\
\hline
\end{tabular}




\begin{tabular}{|c|c|c|c|c|c|c|c|c|c|c|c|c|c|}
\hline & & step3 & 1 & 1.33 & 0.90 & 1.01 & 0.53 & 0.76 & 0.82 & 0.87 & 0.79 & 0.68 & 0.84 \\
\hline \multirow[t]{3}{*}{ spatial } & EMDI & step1 & 1 & 1.30 & 0.77 & 0.94 & 0.58 & 1.05 & 0.74 & 0.77 & 0.76 & 0.97 & 0.79 \\
\hline & & step2 & 1 & 1.30 & 0.80 & 0.96 & 0.59 & 0.89 & 0.75 & 0.72 & 0.76 & 1.00 & 0.77 \\
\hline & & step3 & 1 & 1.30 & 0.91 & 0.87 & 0.58 & 0.66 & 0.79 & 0.83 & 0.81 & 0.65 & 0.80 \\
\hline \multicolumn{14}{|l|}{ LAI } \\
\hline \multirow[t]{3}{*}{ spatial } & MCD15 & step1 & 1 & 1.29 & 0.96 & 0.76 & 0.78 & 0.88 & 0.69 & 1.85 & 0.66 & 1.89 & \\
\hline & & step2 & 1 & 1.29 & 1.07 & 0.83 & 0.73 & 0.74 & 0.67 & 1.73 & 0.58 & 1.63 & \\
\hline & & step3 & 1 & 1.29 & 0.60 & 0.53 & 0.44 & 0.78 & 0.70 & 0.61 & 0.57 & 0.63 & \\
\hline \multirow[t]{3}{*}{ spatial } & AVHRR & step1 & 1 & 1.34 & 1.38 & 1.43 & 1.03 & 0.74 & 0.79 & 2.57 & 0.81 & 2.58 & \\
\hline & & step2 & 1 & 1.34 & 1.54 & 0.90 & 0.80 & 0.74 & 0.69 & 1.77 & 0.60 & 1.67 & \\
\hline & & step3 & 1 & 1.34 & 0.81 & 0.71 & 0.49 & 0.65 & 0.74 & 0.62 & 0.61 & 0.64 & \\
\hline \multicolumn{14}{|c|}{ Carbon in vegetation } \\
\hline spatial & Avitabile & step1 & 1 & 1.32 & 0.73 & 0.89 & 0.89 & 1.35 & 1.30 & 1.27 & 1.30 & 1.27 & 0.73 \\
\hline \multirow{5}{*}{ spatial } & & step2 & 1 & 1.32 & 0.67 & 0.86 & 0.78 & 1.00 & 0.96 & 0.97 & 0.86 & 0.96 & 0.66 \\
\hline & & step3 & 1 & 1.32 & 0.69 & 0.88 & 0.76 & 0.78 & 0.76 & 0.76 & 0.74 & 0.80 & 0.70 \\
\hline & Carvalhais & step1 & 1 & 1.32 & 0.64 & 0.68 & 0.61 & 0.65 & 0.68 & 0.65 & 0.63 & 0.66 & 0.51 \\
\hline & & step2 & 1 & 1.32 & 0.65 & 0.70 & 0.61 & 0.65 & 0.63 & 0.66 & 0.61 & 0.66 & 0.53 \\
\hline & & step3 & 1 & 1.32 & 0.66 & 0.66 & 0.58 & 0.64 & 0.62 & 0.66 & 0.58 & 0.67 & 0.54 \\
\hline
\end{tabular}



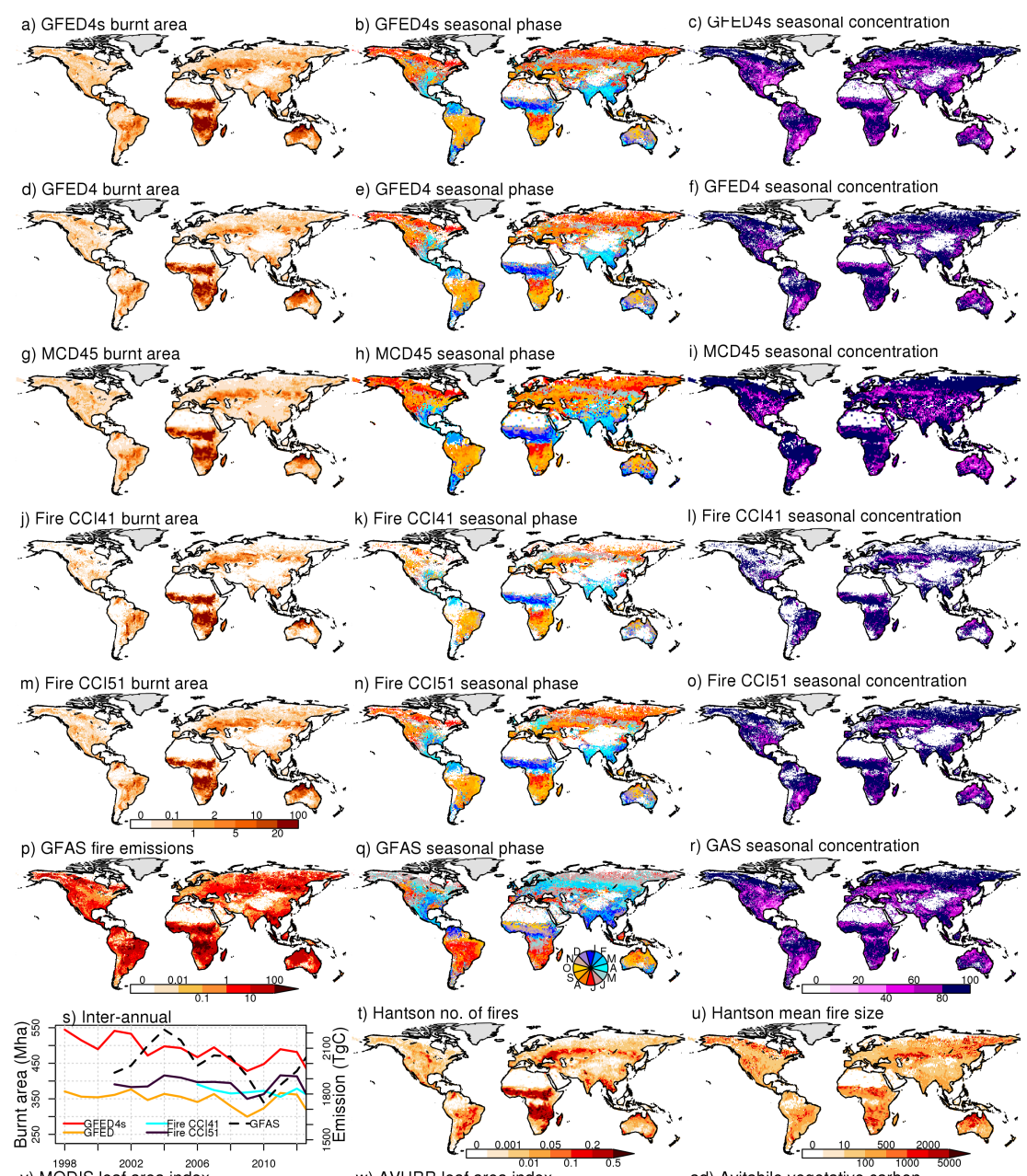

t) Hantson no. of fires

u) Hantson mean fire size
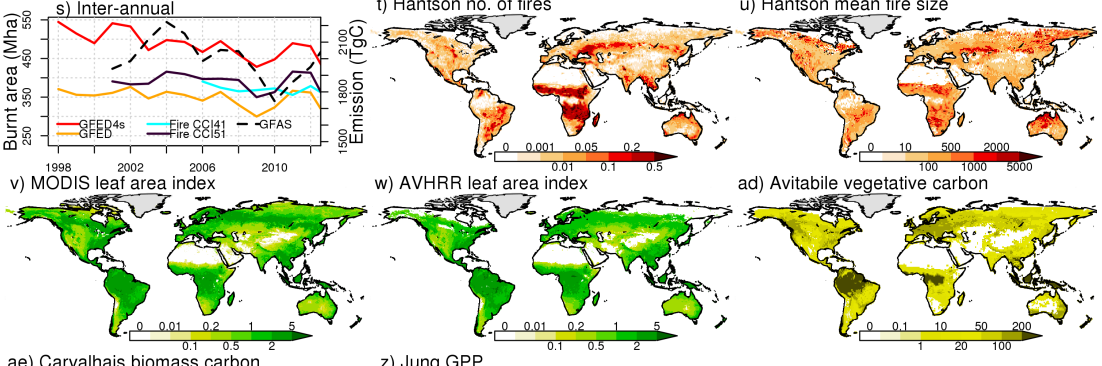

w) AVHRR leaf area index

ad) Avitabile vegetative carbon
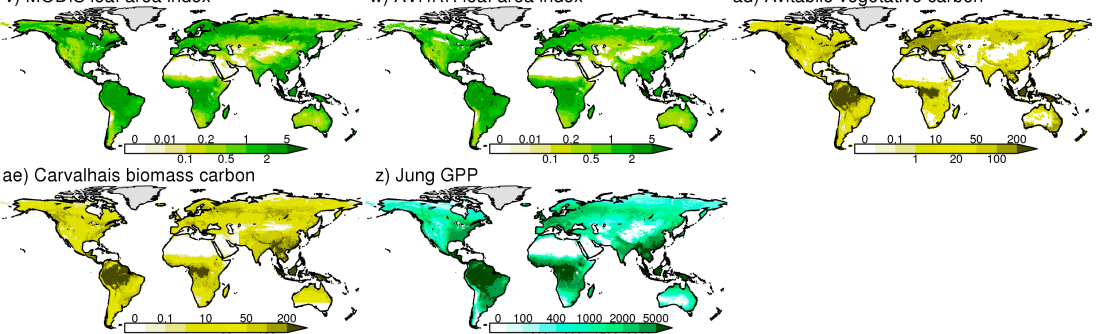

z) Jung GPP
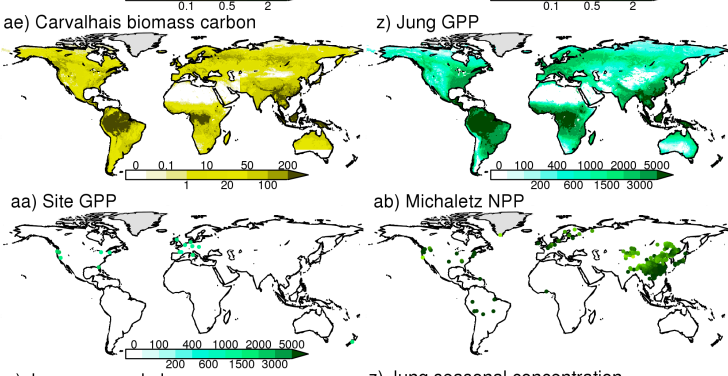

ab) Michaletz NPP

ac) Site NPP
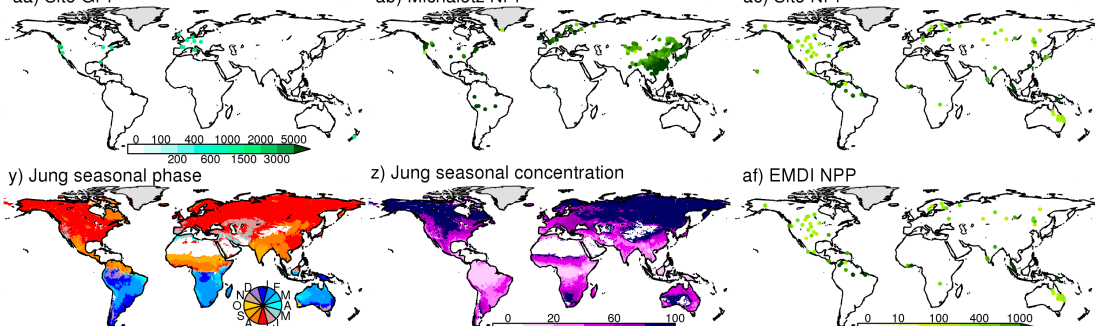

z) Jung seasonal concentration

af) EMDI NPP
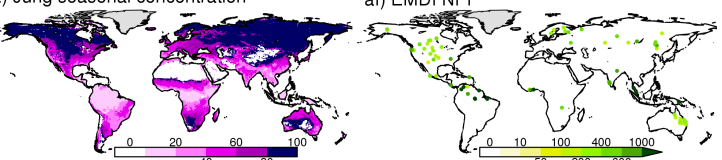

Figure S1: Overview of the reference datasets used. 


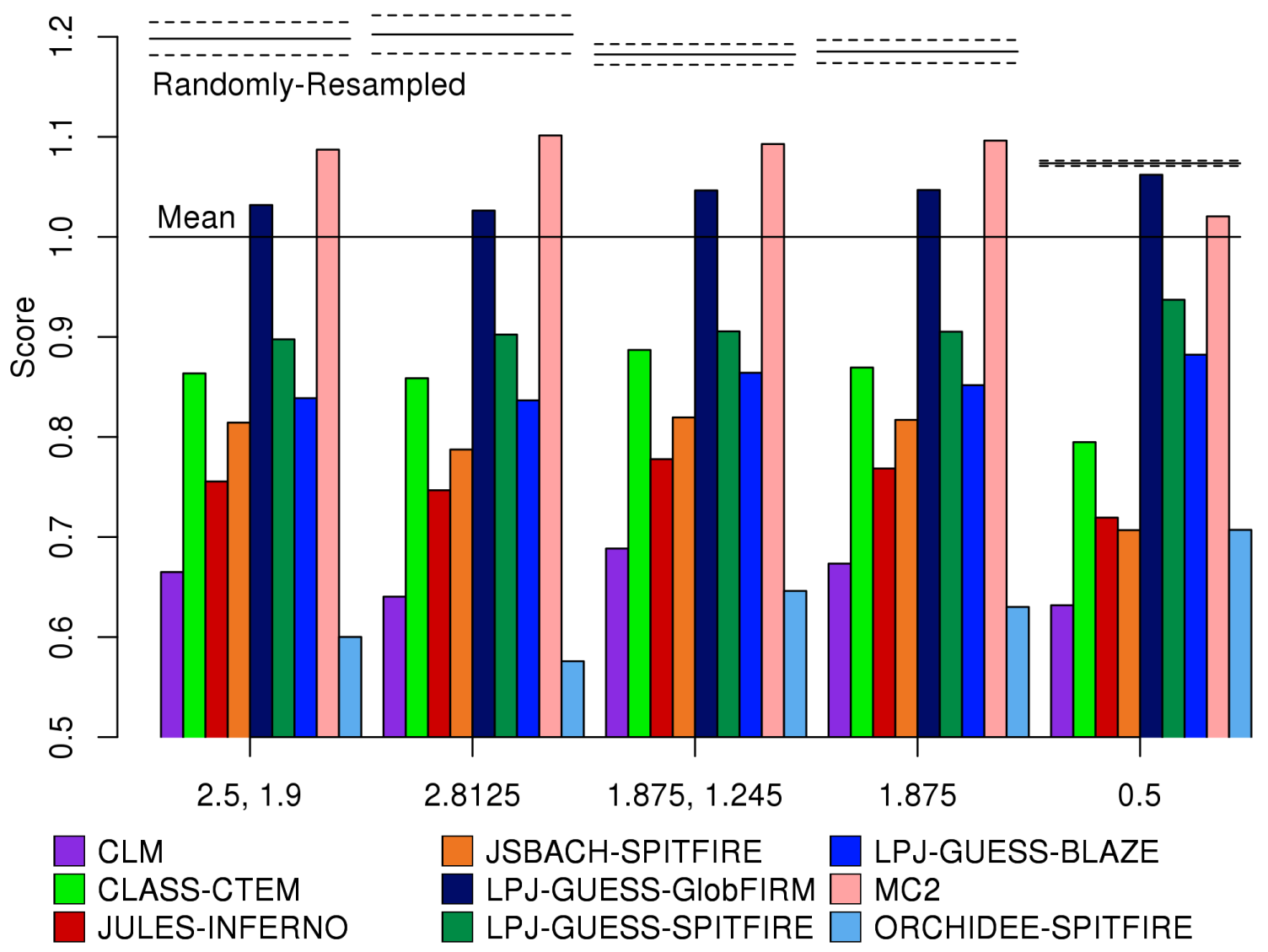

Figure S2: Benchmark scores for each model compared against GFED4s burnt area step 3 at different resolutions for CLM, CLASSCTEM, JULES-inferno, JSBACH, LPJ-GUESS-GlobFIRM, LPJ-GUESS-SPITFIRE, LPJ-GUESS-BLAZE, MC2 and ORCHIDEE respectively. Each block (left to right) shows the comparison conducted by resampling model output and GFED4s to the $2.5 \mathrm{x} 1.9^{\circ}$ grid of CLM; $2.8125 \times 2.8125^{\circ}$ CLASS-CTEM grid; $1.875 \times 1.25^{\circ}$ JULES-INFERNO grid, $1.875 \times 1.875^{\circ}$ JSBACH-SPITFIRE grid; and the $0.5 \times 0.5^{\circ}$ grid used by all other models and for the benchmarking. 